\title{
MRS Elects Officers, Councillors for 2000
}

Members of the Materials Research Society have elected two officers and three councillors to join the 2000 Council, which consists of the Executive Committee and 15 councillors. The annual election ended October 15, 1999.

\section{MRS Officers}

President

Harry A. Atwater (2000)

California Institute of Technology

Immediate Past President

Ronald Gibala (2000)

University of Michigan

Vice President (President-Elect)

*Marty Green (2000)

Bell Laboratories, Lucent Technologies

Secretary

*Chuang Chuang Tsai (2001)

Applied Komatsu Technology

Treasurer

Merrilea J. Mayo (2000)

Pennsylvania State University
2000 MRS Councillors

Michael J. Aziz (2000)

Harvard University

*Orlando Auciello (2002)

Argonne National Laboratory

Katayun Barmak (2000)

Carnegie Mellon University

John W. Cahn (2001)

National Institute of Standards and Technology

Anthony K. Cheetham (2001)

University of California-Santa Barbara

David J. Eaglesham (2000)

Bell Laboratories, Lucent Technologies

A. Lindsay Greer (2001)

Cambridge University

Alexander H. King (2000)

Purdue University

Karen Maex (2000)

IMEC, Belgium
Stephen J. Pennycook (2001)

Oak Ridge National Laboratory

Caroline A. Ross (2001)

Massachusetts Institute of Technology

Alan I. Taub (2000)

Ford Motor Company

Rudolf M. Tromp (2001)

IBM T.J. Watson Research Center

${ }^{*}$ R. Stanley Williams (2002)

Hewlett-Packard Laboratories

${ }^{*}$ Anne M. Mayes (2002)

Massachusetts Institute of Technology

(Terms of office expire at the end of the years indicated in parentheses.)

${ }^{*}$ Newly elected.

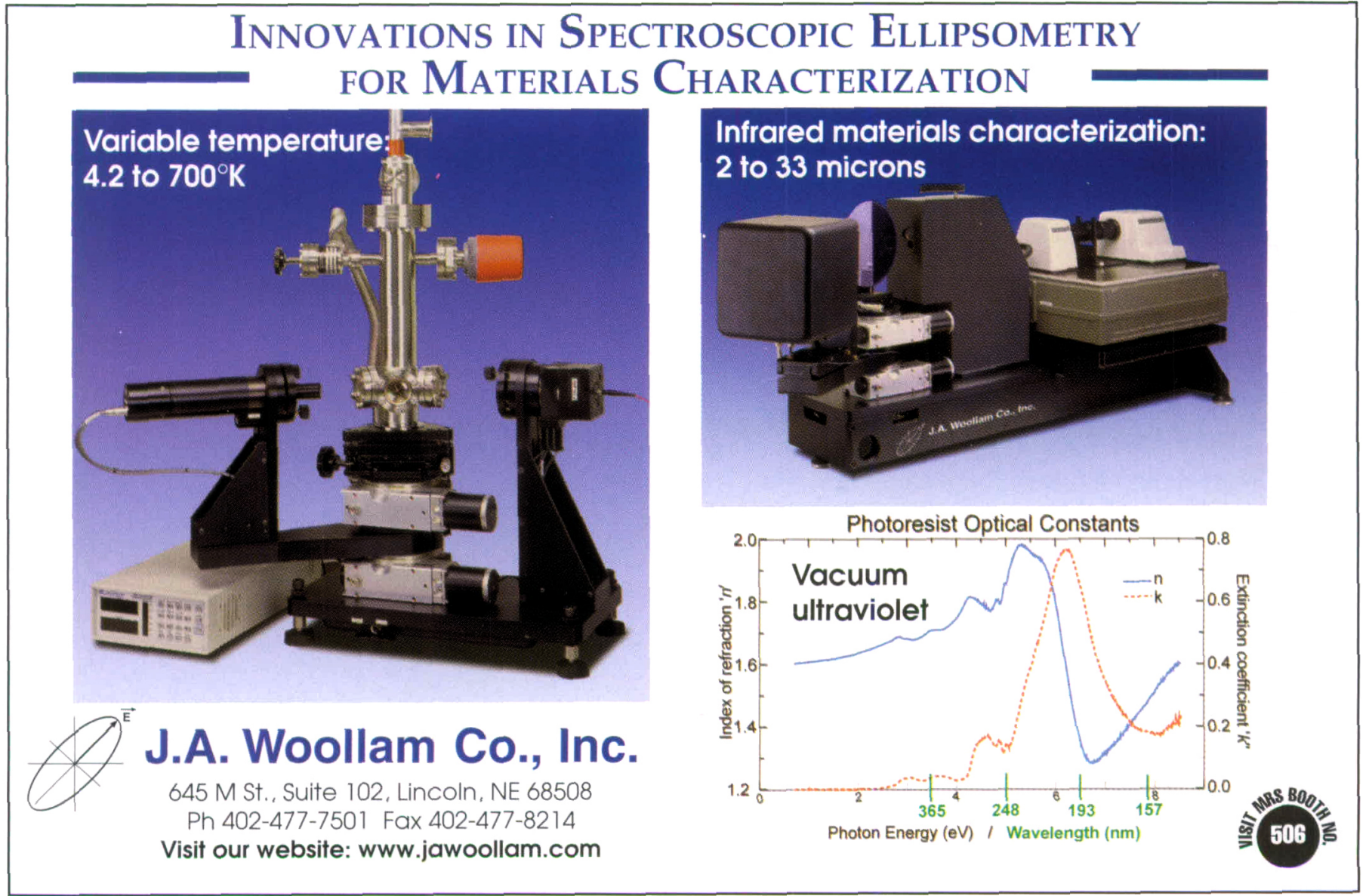


The MRS Exhibit, held in conjunction with the 1999 MRS Fall Meeting, will feature more than 225 international exhibitors from all sectors of the materials science and engineering marketplace. Learn the latest techniques, advances and future of materials science and engineering directly from the manufacturers, suppliers and developers. Or browse the new releases, publications and journals from the various publishers. The exhibit will closely parallel the nature of the technical symposia and, as always, the program has been arranged to allow meeting participants ample opportunity to visit the exhibit.

\section{Exhibit Hours}

\begin{tabular}{lr}
\hline Tuesday, November 30 & $11: 30 \mathrm{am}-6: 00 \mathrm{pm}$ \\
\hline Wednesday, December 1 & 9:00 am - 6:00 pm \\
\hline Thursday, December 2 & $9: 00 \mathrm{am}-2: 00 \mathrm{pm}$
\end{tabular}

The 1999 MRS Fall Exhibit-everything you need... all under one roof!

Partial List of Fall Exhibitors

\section{Products \& Services}

A \& N Corporation

ABB Extrel

Advanced Control Systems Corporation

Advanced Research Systems, Inc.

aixACCT

AIXTRON, Inc.

AJA International, Inc.

Akzo Nobel Chemicals Inc.

Alcatel Vacuum Products, Inc.

Aldrich Chemical Company, Inc.

Alfa Aesar, a Johnson Matthey Company

Altran Corporation

American Superconductor

Andeen-Hagerling, Inc.

Angstrom Sciences, Inc.

Applied Nano Metrics, Inc.

Applied Surface Technologies

Avanti Polar Lipids, Inc.

Bede Scientific Incorporated

Bicron Crystal Products

Bio-Rad, Spectroscopy Division

Bioanalytical Systems, Inc. (BAS)

Blake Industries, Inc.

BOC Edwards

Bruker AXS, Inc.

Buehler, Ltd

Burleigh Instruments, Inc

Caburn-International, Inc.

Cameca Instruments, Inc.

Capovani Brothers Inc.

Center For Tribology, Inc.

CERAC, inc.

Ceramaseal

Chemat Technology, Inc.

Chemlcon Inc.

Clemex Technologies, Inc.

Commonwealth Scientific

Cree Research, Inc

Cressington Scientific Instruments

CRI, Inc.

CRYO Industries of America, Inc.

Cryogenic Control Systems, Inc.

Cryomech, Inc.

DCA Instruments, Inc.

Del Electronics,

Power Conversion Products

Denton Vacuum LLC

Digital Instruments,

Veeco Metrology Group

Duniway Stockroom Corporation

Eagle-Picher Technologies, LLC

EDAX Inc.

EG\&G Instruments, Inc

EMCORE Corporation

EOS-EyeOnScience

EPI MBE Products Group

Epichem Inc.
Epion Corporation

ESCETE Single Crystal Technology B.V.

ESM Software

Evans Analytical Group

EXAKT Technologies, Inc.

FEI Company/Philips Electron Optics

Fischione Instruments

FRT of America

Fujikin of America, Inc.

Genus, Inc.

GFS Chemicals, Inc

Goodfellow Corporation

Hamamatsu Corporation

High Voltage Engineering Europa B.V.

Hinds Instruments, Inc.

Hitachi Scientific Instruments

Huntington Mechanical Laboratories, Inc.

Hysitron, Inc.

Implant Sciences Corporation

Industrial Science \& Technology

Network, Inc.

Inel, Inc.

Innovative Technology, Inc.

Inorgtech Ltd.

INSACO, Inc.

Institution of Electrical Engineers/INSPEC

Instron Corporation

Instruments S.A., Inc./

Horiba Group/Raman Division

Insulator Seal, Inc.

Ion Tech, Inc.

IXRF Systems, Inc.

Janis Research Company, Inc.

JCPDS-ICDD

JEOL USA, Inc.

J.I.P.ELEC

Jobin Yvon/HORIBA

Johnsen Ultravac

Johnson Matthey Electronics

k-Space Associates, Inc.

Keithley Instruments, Inc.

Kimball Physics, Inc.

KLA-Tencor Corporation

Kratos Analytical Inc.

Kurt J. Lesker Company

Ladd Research Industries, Inc.

Lake Shore Cryotronics, Inc.

Lambda Physik, Inc.

Laser Technology West, LLC

LEO Electron Microscopy Inc.

Leybold Vacuum Products, Inc.

Logitech Product Group

Materials Preparation Center/

Ames Laboratory U.S. DOE

MBRAUN, Inc.

MDC Vacuum Products Corporation

Micro Photonics Inc.

Microcal Software, Inc.

MKS Instruments, Inc.

MMR Technologies, Inc.
Molecular Metrology, Inc.

Molecular Simulations Inc

MTI Corporation

MTS Systems Corporation

Nanomics Imaging Ltd.

National Aeronautics \& Space

Administration, Lyndon B. Johnson

Space Center

National Electrostatics Corp.

Neocera, Inc.

New Wave Research, Inc.

NFT-Nanofilm Technologie GmbH

Nicolet Instrument Corporation

NORAN Instruments Inc.

NYS Center for Advanced Thin Film Technology

Olympus America Inc.

Omicron Associates

Oriel Instruments

Osmic, Inc.

Oxford Applied Research

Oxford Instruments America, Inc.

Microanalysis Group

Oxford Instruments America, Inc./

Research Instruments Division

Ted Pella, Inc.

Performance Materials

Perkin Elmer

Pfeiffer Vacuum

Philips Analytical

Physical Electronics

PIEZOMAX Technologies, Inc.

Polaroid Corporation,

Digital Products

Polytec PI, Inc.

Praxair Specialty Ceramics

Princeton Gamma-Tech, Inc.

Princeton Scientific Corp.

Proxitronics/Stanford Computer Optics

Pure Tech, Subsidiary of Williams

Advanced Materials, Inc.

Quad Group, Inc.

Quantum Design, Inc.

Quesant Instrument Corporation

Radiant Technologies, Inc.

Refining Systems, Inc.

Renishaw Inc.

Research and PVD Materials Corporation

Reveo, Inc.

Rheometric Scientific, Inc.

RHK Technology, Inc.

Riber, Inc.

Rigaku/USA, Inc.

RJ Lee Instruments Limited

Rohm and Haas Company

RONTEC USA, InC.

Royal Society of Chemistry

Schott Glass Technologies Inc.

Scintag, Inc.

SciVision

SELA USA, Inc.
Sigma Instruments

Silicon-MDT Ltd.

Silicon Sense, Inc.

SKION/InnoVac Corporation

Solartron inc.

SOPRA, Inc.

South Bay Technology, Inc

SPECS USA, Inc.

SPI Supplies

STAIB Instruments, Inc.

Sterling Semiconductor, Inc.

Strem Chemicals, Inc.

Structured Materials Industries, Inc./

Nanopowder Enterprises Inc.

Superconductive Components, Inc.

SURFACE

Surface/Interface, Inc.

SVT Associates, Inc

Sycon Instruments, Inc.

TA Instruments, Inc.

Telemark

Textron Systems

M. Theiss Hard- and Software

Thermionics Vacuum Products

TherMold Partners, LP

ThermoMicroscopes

Thomas Swan Scientific Equipment Ltd

TORR International, Inc.

TPL, Inc.

Twente Solid State Technology B.V.

Universal Voltronics Corporation

Vacuum Atmospheres Company

Vacuum Research Corporation

Varian Vacuum Technologies

VAT, Inc.

VG Scientific

Virginia Semiconductor, Inc.

R.D. Webb Company

Well Diamond Wire Saws, Inc.

J.A. Woollam Co., Inc.

X-Ray Optical Systems, Inc.

Zygo Corporation

\section{Publishers}

Academic Press, Inc.

American Chemical Society

American Institute of Physics

American Physical Society

Cambridge University Press

Elsevier Science

Gordon \& Breach (IPD)/

Harwood Academic

Institute of Physics Publishing

Kluwer Academic/Plenum Publishers

Oxford University Press

Springer-Verlag New York, Inc.

Taylor \& Francis

John Wiley \& Sons, Inc.

See Ad In This Issue 


\begin{tabular}{|c|c|c|c|}
\hline \multicolumn{4}{|c|}{ Advertisers in This Issue } \\
\hline & Page No. & & Page No. \\
\hline "Academic Press, Inc. & 74 & *JEOL USA, InC. & 10 \\
\hline *Aldrich Chemical Company, Inc. & $\overline{69}$ & ${ }^{\star}$ MDC Vacuum Products Corporation & 50 \\
\hline Andeen-Hagerling, Inc. & 61 & * National Electrostatics Corp. & 39 \\
\hline Australian Scientific Instruments Pty Ltd & 17 & ${ }^{\star}$ Osmic, Inc. & 63 \\
\hline * Bede Scientific Incorporated & Inside back cover & Oxford Cryosystems & 18 \\
\hline "Cambridge University Press & 72 & ${ }^{*}$ Oxford University Press & 78 \\
\hline * Chemat Technology, Inc. & $\overline{12}$ & *Philips Analytical & 1 \\
\hline * Digital Instruments & 2 & *Princeton Gamma-Tech, Inc. & 8 \\
\hline "EDAX Inc. & 16 & ${ }^{*}$ Quad Group, Inc. & 7 \\
\hline *Elsevier Science & 77 & ${ }^{*}$ Quesant Instrument Corporation & 26 \\
\hline *ESM Software & 13 & Saint-Gobain Industrial Ceramics, Inc. & 9 \\
\hline${ }^{*}$ Fischione Instruments & $\overline{11}$ & ${ }^{*}$ Springer-Verlag New York, Inc. & 73 \\
\hline GMW & 6 & ${ }^{*}$ Thermionics Vacuum Products & 56 \\
\hline *Goodfellow Corporation & 32 & *VAT, Inc. & 44 \\
\hline * Gordon \& Breach & 76 & *Virginia Semiconductor, Inc. & 14 \\
\hline * High Voltage Engineering & Inside front cover & Voltaix, Inc. & 66 \\
\hline * Huntington Mechanical Laboratories, Inc. & Outside back cover & *J.A. Woollam Co., Inc. & 64 \\
\hline${ }^{*}$ Hysitron, Inc. & 15,71 & & \\
\hline \multicolumn{4}{|c|}{$\begin{array}{l}\text { For free information about the products and services offered in this issue, check www. mrs.org/publications/ bulletin/advertisers } \\
\text { or fill out and mail the Reader Sevvice Card, or FAX it to 312-922-3165. }\end{array}$} \\
\hline \multicolumn{4}{|c|}{ *Please visit us at the Exhibit, November 30-December 2, during the 1999 MRS Fall Meeting in Boston. } \\
\hline
\end{tabular}

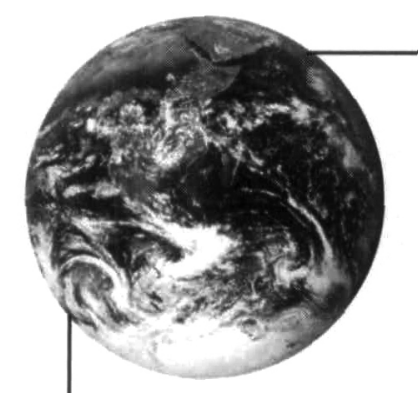

PRODUCTS

Silane

Disilane

Trisilane

Difluorosilane

Trifluorosilane

Silicon Tetrafluoride

Germane

Digermane

Germanium Tetrafluoride

Methylsilane

Diborane

Boron Trifluoride

Trimethylboron

Phosphine

Deuterated Silane

Deuterated Germane

Deuterated Methane

Deuterated Diborane

Deuterated Trimethylboron

\section{Voliaix, Inc.}

Gases for Research and Manufacturing of Silicon-based Semiconductors

- ISOTOPICALLY ENRICHED

${ }^{11} \mathrm{BF}_{3} \cdot{ }^{10} \mathrm{BF}_{3} \cdot{ }^{29} \mathrm{SiF}_{4} \cdot{ }^{11} \mathrm{~B}\left(\mathrm{CH}_{3}\right)_{3} \cdot{ }^{11} \mathrm{~B}_{2} \mathrm{H}_{6} \cdot$ Deuterated variants

- UV PHOTORESIST \& FLOWABLE DIELECTRIC PRECURSORS Methylsilane

- NEXT GENERATION SILICON PRECURSORS

Disilane (a liquified gas) - Trisilane (a liquid source)

Trifluorosilane (for faster dielectrics)

Difluorosilane (for silicon deposition at lower temperature)

- SILICON ALLOY PRECURSORS Germane $\cdot$ Digermane Methylsilane

- ADVANCED DOPANTS Trimethylboron
For more information about Voltaix products and services contact us.

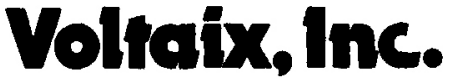

PO Box 5357, North Branch, NJ 08876, USA Tel: 908 231-9060 - Fax: 908 231-9063 Toll-Free: 1-800-VOLTAIX in USA \& CANADA

\section{EXPLORE THE VOLTAIX WEB SITE WWW.voltaix.com}

